\title{
Relative Smooth Topological Spaces
}

\author{
B. Ghazanfari \\ Department of Mathematics, Faculty of Science, Lorestan University, P.O. Box 465, Khoramabad 68137-17133, Iran
}

Correspondence should be addressed to B. Ghazanfari, bahman_ghazanfari@yahoo.com

Received 22 June 2009; Accepted 10 November 2009

Recommended by Zne-Jung Lee

In 1992, Ramadan introduced the concept of a smooth topological space and relativeness between smooth topological space and fuzzy topological space in Chang's (1968) view points. In this paper we give a new definition of smooth topological space. This definition can be considered as a generalization of the smooth topological space which was given by Ramadan. Some general properties such as relative smooth continuity and relative smooth compactness are studied.

Copyright ( 2009 B. Ghazanfari. This is an open access article distributed under the Creative Commons Attribution License, which permits unrestricted use, distribution, and reproduction in any medium, provided the original work is properly cited.

\section{Introduction}

Let $X$ be a nonempty set and let $\mathcal{L}, \mathcal{L}^{\prime}$ be two lattice which will be copies of $[0,1]$ or $\{0,1\}$. The family of all fuzzy sets on $X$ will be denoted by $\mathcal{L}^{X}$ Zadeh [1].

In the consideration of the nature an observer can be modeled by an operator which evaluates each proposition by a number in the closed interval $[0,1]$; see Anvari and Molaei [2] and Molaei [3]. We assume that $\mu$ as a function from $X$ to $\mathcal{L}$ is an observer of $X$ on lattice $\mathcal{L}$ and denote $[\mu]=\left\{\lambda \in \mathcal{L}^{X}: \lambda \subseteq \mu\right\}$, where $\lambda \subseteq \mu$ implies that $\lambda(x) \leq \mu(x)$ for all $x \in X$.

Definition 1.1. Let $\mu \in \mathcal{L}^{X}$. A relative smooth topological space or $\mu$-smooth topological space or $\mu$-STS for short is a triple $\left(X, \mu, \mathcal{T}_{\mu}\right)$, where $\mathcal{T}_{\mu}:[\mu] \rightarrow \mathcal{L}^{\prime}$ is a mapping satisfying the following properties:

(i) $\mathcal{T}_{\mu}(\mu)=\mathcal{T}_{\mu}\left(\chi_{\phi}\right)=1$, where $\chi$ is the characteristic function;

(ii) if $\lambda_{1}, \lambda_{2} \in[\mu]$, then $\mathcal{T}_{\mu}\left(\lambda_{1} \cap \lambda_{2}\right) \geq \mathcal{T}_{\mu}\left(\lambda_{1}\right) \wedge \mathcal{T}_{\mu}\left(\lambda_{2}\right)$, where $\wedge$ is the minimum operator in $\mathcal{L}^{\prime}$;

(iii) $\mathcal{T}_{\mu}\left(\cup\left\{\lambda_{i}: i \in I\right\}\right) \geq \wedge\left\{\mathcal{T}_{\mu}\left(\lambda_{i}\right): i \in I\right\}$.

We call $\mathcal{T}_{\mu}$ a smooth topology from view point of $\mu$ or a $\mu$-smooth topology or a fuzzy family of $\mu$-open sets on $X$.

Remark 1.2. If $\mu=\chi_{X}$ then the $\chi_{X}$-STS $\left(X, \chi_{X}, \mathcal{T}_{\chi_{X}}\right)$ coincides with the smooth topological space $(X, \tau)$ defined by Ramadan [4], and if we take $\mathcal{L}=[0,1], \mathcal{L}^{\prime}=\{0,1\}$, and
$\mu=\chi_{X}$ then the $\chi_{X}$-STS coincides with the known definition of fuzzy topological space $(\bar{X}, \tau)$ defined by Chang [5]. If $\mathcal{L}=\mathcal{L}^{\prime}=\{0,1\}$, and $\mu=\chi_{X}$ then $\mathcal{T}_{\chi_{X}}$ is a classical topology.

Definition 1.3. Let $\mu \in \mathcal{L}^{X}$. A $\mu$-smooth cotopological space is a triple $\left(X, \mu, \mathcal{F}_{\mu}\right)$, where $\mathcal{F}_{\mu}:[\mu] \rightarrow \mathcal{L}^{\prime}$ is a mapping satisfying the following properties:

(i) $\mathcal{F}_{\mu}(\mu)=\mathcal{F}_{\mu}\left(\chi_{\phi}\right)=1$;

(ii) if $\eta_{1}, \eta_{2} \in[\mu]$, then $\mathcal{F}_{\mu}\left(\eta_{1} \cup \eta_{2}\right) \geq \mathcal{F}_{\mu}\left(\eta_{1}\right) \wedge \mathscr{F}_{\mu}\left(\eta_{2}\right)$;

(iii) $\mathcal{F}_{\mu}\left(\cap\left\{\eta_{i}: i \in I\right\}\right) \geq \wedge\left\{\mathcal{F}_{\mu}\left(\eta_{i}\right): i \in I\right\}$.

We call $\mathcal{F}_{\mu}$ a $\mu$-smooth co-topology or a fuzzy family of $\mu$-closed sets on $X$.

Theorem 1.4. Let $\left(X, \mu, \mathcal{T}_{\mu}\right)$ be a $\mu$-STS and $\mathcal{F}_{\mu}:[\mu] \rightarrow \mathcal{L}^{\prime}$ be a mapping defined by $\mathcal{F}_{\mu}(\eta)=\mathcal{T}_{\mu}\left(\eta^{\prime}\right)$, where $\eta^{\prime}=\mu-\eta$. Then $\mathcal{F}_{\mu}$ is a fuzzy family of $\mu$-closed sets.

Proof.

(i) It is clear.

(ii) It flows from

$$
\begin{gathered}
\left(\eta_{1} \cup \eta_{2}\right)^{\prime}=\mu-\left(\eta_{1} \cup \eta_{2}\right)=\mu-\sup \left\{\eta_{1}, \eta_{2}\right\} \\
=\inf \left\{\mu-\eta_{1}, \mu-\eta_{2}\right\}=\eta_{1}^{\prime} \cap \eta_{2}^{\prime}
\end{gathered}
$$


(iii) It flows from

$$
\begin{aligned}
\left(\cap_{i \in I} \eta_{i}\right)^{\prime} & =\mu-\cap_{i \in I} \eta_{i}=\mu-\inf \left\{\eta_{i}: i \in I\right\} \\
& =\sup \left\{\mu-\eta_{i}: i \in I\right\}=\cup_{i \in I} \eta_{i}^{\prime} .
\end{aligned}
$$

So, $\mathcal{F}_{\mu}\left(\cap\left\{\eta_{i}: i \in I\right\}\right)=\mathcal{T}_{\mu}\left(\cup\left\{\eta_{i}^{\prime}: i \in I\right\}\right)$.

Theorem 1.5. Let $\mathcal{F}_{\mu}$ be a fuzzy family of $\mu$-closed sets and define $\mathcal{T}_{\mathcal{F}_{\mu}}:[\mu] \rightarrow \mathcal{L}^{\prime}$ by $\mathcal{T}_{\mathcal{F}_{\mu}}(\eta)=\mathcal{F}_{\mu}\left(\eta^{\prime}\right)$. Then $\mathcal{T}_{\mathcal{F}_{\mu}}$ is a $\mu$-STS on $X$.

Proof. The proof is similar to the previous theorem.

Corollary 1.6. Let $\mathcal{T}_{\mu}$ be a $\mu$-STS and $\mathcal{F}_{\mu}$ a fuzzy family of $\mu$-closed sets. Then $\mathcal{T}_{\mathcal{F}_{\mathcal{F}_{\mu}}=\mathcal{T}_{\mu}}$ and $\mathcal{F}_{\mathcal{T}_{\mathcal{F}_{\mu}}=\mathcal{F}_{\mu}}$.

Proof. Suppose $\lambda, \eta \in[\mu]$ then we have $\mathcal{T}_{\mathcal{F}_{\mathcal{T}_{\mu}}}(\lambda)=\mathcal{F}_{\mathcal{T}_{\mu}}\left(\lambda^{\prime}\right)=$ $\mathcal{T}_{\mu}(\lambda)$ and $\mathcal{F}_{\mathcal{T}_{\mathcal{F}_{\mu}}}(\eta)=\mathcal{T}_{\mathcal{F}_{\mu}}\left(\eta^{\prime}\right)=\mathcal{F}_{\mu}(\eta)$.

Example 1.7. Let $X$ be the set of all differentiable real-valued functions on $(1, \infty)$, with positive derivative of order one and let $\mathcal{L}$ be the set of real-valued functions defined on $(1, \infty)$. Let $\mu: X \rightarrow \mathcal{L}$ be defined by $\mu(f)=f^{\prime}+$ Exp, where Exp is the exponential function. For nonnegative integer $n$ define $\lambda^{n}: X \rightarrow \mathcal{L}$ by

$$
\left(\lambda^{n}(f)\right)(x)=f^{\prime}(x)+\sum_{i=1}^{n} \frac{x^{i-1}}{(i-1) !} .
$$

If we take $\mathcal{L}^{\prime}=[0,1]$ and define $\mathcal{T}_{\mu}:[\mu] \rightarrow[0,1]$ by $\mathcal{T}_{\mu}\left(\chi_{\varnothing}\right)=\mathcal{T}_{\mu}(\mu)=1 ; \mathcal{T}_{\mu}\left(\lambda^{n}\right)=1-1 / n$ for $n=1,2, \ldots$ Then $\left(X, \mu, \mathcal{T}_{\mu}\right)$ is a $\mu$-STS. Since $\lambda^{n} \cap \lambda^{m}=\lambda^{m}, \lambda^{n} \cup \lambda^{m}=\lambda^{n}$, where $n>m$ and $\cup \lambda^{n_{i}}=\mu$ whenever $n_{i}$ tends to $+\infty$, so

$$
\mathcal{T}_{\mu}\left(\lambda^{n} \cap \lambda^{m}\right)=\mathcal{T}_{\mu}\left(\lambda^{m}\right) \geq \mathcal{T}_{\mu}\left(\lambda^{n}\right) \wedge \mathcal{T}_{\mu}\left(\lambda^{m}\right),
$$

and for $I \subseteq \mathbb{N}$ we find

$$
\widetilde{\mathcal{T}}_{\mu}\left(\cup\left\{\lambda^{n}: n \in I\right\}\right) \geq \wedge\left\{\mathcal{T}_{\mu}\left(\lambda^{n}\right): n \in I\right\} .
$$

Definition 1.8. Let $\mathcal{T}_{\mu}^{1}$ and $\mathcal{T}_{\mu}^{2}$ be two $\mu$-smooth topological spaces on $X$. We say that $\mathcal{T}_{\mu}^{1}$ is finer than $\mathcal{T}_{\mu}^{2}$ or $\mathcal{T}_{\mu}^{2}$ is coarser than $\mathcal{T}_{\mu}^{1}$ and denoted by $\mathcal{T}_{\mu}{ }^{1} \succ \mathcal{T}_{\mu}{ }^{2}$ if $\mathcal{T}_{\mu}{ }^{1}(\lambda) \geq \mathcal{T}_{\mu}{ }^{2}(\lambda)$ for every $\lambda \in[\mu]$.

Theorem 1.9. Let $\left\{\mathcal{T}_{\mu}^{i}: i \in I\right\}$ be a family of $\mu$-STS on $X$. Then $\mathcal{T}_{\mu}=\wedge_{i \in I} \mathcal{T}_{\mu}^{i}$ is also $\mu$-STS on $X$, where

$$
\left(\wedge_{i \in I} \mathcal{T}_{\mu}^{i}\right)(\lambda)=\wedge_{i \in I} \mathcal{T}_{\mu}^{i}(\lambda) .
$$

Proof.

(i) It is clear.

(ii) For every $\lambda, \eta \in[\mu]$,

$$
\begin{aligned}
\mathcal{T}_{\mu}(\lambda \cap \eta) & =\wedge_{i \in I} \mathcal{T}_{\mu}^{i}(\lambda \cap \eta) \geq \wedge_{i \in I}\left(\mathcal{T}_{\mu}^{i}(\lambda) \wedge \mathcal{T}_{\mu}^{i}(\eta)\right) \\
& =\wedge_{i \in I}\left(\mathcal{T}_{\mu}^{i}(\lambda)\right) \wedge\left(\wedge_{i \in I} \mathcal{T}_{\mu}^{i}(\lambda)\right) \\
& =\mathcal{T}_{\mu}(\lambda) \wedge \mathcal{T}_{\mu}(\eta) .
\end{aligned}
$$

(iii) For $\Gamma$,

$$
\begin{aligned}
\mathcal{T}_{\mu}\left(\cup\left\{\lambda_{j}: j \in \Gamma\right\}\right) & =\wedge_{i \in I} \mathcal{T}_{\mu}^{i}\left(\cup\left\{\lambda_{j}: j \in \Gamma\right\}\right) \\
& \geq \wedge_{i \in I} \wedge\left\{\mathcal{T}_{\mu}^{i}\left(\lambda_{j}\right): j \in \Gamma\right\} \\
& =\wedge\left(\wedge_{i \in I}\left\{\mathcal{T}_{\mu}^{i}\left(\lambda_{j}\right): j \in \Gamma\right\}\right) \\
& =\wedge\left\{\mathcal{T}_{\mu}\left(\lambda_{j}\right): j \in \Gamma\right\} .
\end{aligned}
$$

Let $A$ be a subset of $X$ and $\lambda \in[\mu]$. The restriction of $\lambda$ on $A$ is denoted by $\left.\lambda\right|_{A}$.

Theorem 1.10. Let $\left(X, \mu, \mathcal{T}_{\mu}\right)$ be a $\mu$-STS and $A \subset X$. Define a mapping $\mathcal{T}_{\left.\mu\right|_{A}}:[\mu] \rightarrow \mathcal{L}^{\prime}$ by $\mathcal{T}_{\left.\mu\right|_{A}}(\lambda)=\vee\left\{\mathcal{T}_{\mu}(\eta): \eta \in\right.$ $\left.[\mu],\left.\eta\right|_{A}=\lambda\right\}$. Then $\mathcal{T}_{\left.\mu\right|_{A}}$ is a $\mu$-STS on $A$.

Proof.

(i) It is clear that $\mathcal{T}_{\left.\mu\right|_{A}}\left(\chi_{\varnothing}\right)=\mathcal{T}_{\left.\mu\right|_{A}}(\mu)=1$.

(ii) $\forall \lambda_{1}, \lambda_{2} \in \mathscr{L}^{A}, \lambda_{1}, \lambda_{2} \subset \mu$.

$$
\begin{aligned}
\mathcal{T}_{\left.\mu\right|_{A}}\left(\lambda_{1}\right) \wedge \mathcal{T}_{\left.\mu\right|_{A}}\left(\lambda_{2}\right) \\
=\vee\left\{\mathcal{T}_{\mu}\left(\eta_{1}\right): \eta_{1} \in[\mu],\left.\eta_{1}\right|_{A}=\lambda_{1}\right\} \\
\wedge \vee\left\{\mathcal{T}_{\mu}\left(\eta_{2}\right): \eta_{2} \in[\mu],\left.\eta_{2}\right|_{A}=\lambda_{2}\right\} \\
=\vee\left\{\mathcal{T}_{\mu}\left(\eta_{1}\right) \wedge \mathcal{T}_{\mu}\left(\eta_{2}\right): \eta_{1}, \eta_{2} \in[\mu],\left.\eta_{1} \cap \eta_{2}\right|_{A}\right. \\
\left.\quad=\lambda_{1} \cap \lambda_{2}\right\} \\
\leq \vee\left\{\mathcal{T}_{\mu}\left(\eta_{1} \cap \eta_{2}\right): \eta_{1}, \eta_{2} \in[\mu],\left.\eta_{1} \cap \eta_{2}\right|_{A}=\lambda_{1} \cap \lambda_{2}\right\} \\
=\mathcal{T}_{\left.\mu\right|_{A}}\left(\lambda_{1} \cap \lambda_{2}\right) .
\end{aligned}
$$

(iii) $\forall i \in I, \mathcal{T}_{\left.\mu\right|_{A}}\left\{\lambda_{i}: i \in I\right\}=\vee\left\{\mathcal{T}_{\mu}\left(\eta_{i}\right): \eta_{i} \in[\mu],\left.\eta_{i}\right|_{A}=\right.$ $\left.\lambda_{i}\right\}$. So

$$
\begin{aligned}
\mathcal{T}_{\left.\mu\right|_{A}} & \left(\cup\left\{\lambda_{i}: i \in I\right\}\right) \\
& =\vee\left\{\mathcal{T}_{\mu}\left(\cup\left\{\eta_{i}: i \in I\right\}\right): \eta_{i} \in[\mu],\left.\cup \eta_{i}\right|_{A}=\cup \lambda_{i}\right\} \\
& \leq \vee\left\{\wedge\left\{\mathcal{T}_{\mu}\left(\eta_{i}\right): i \in I\right\}: \eta_{i} \in[\mu],\left.\cup \eta_{i}\right|_{A}=\cup \lambda_{i}\right\} \\
& =\wedge \mathcal{T}_{\left.\mu\right|_{A}}\left\{\lambda_{i}: i \in I\right\} .
\end{aligned}
$$

Definition 1.11. The $\mu$-STS $\left(A, \mu, \mathcal{T}_{\left.\mu\right|_{A}}\right)$ is called a subspace of $\left(X, \mu, \mathcal{T}_{\mu}\right)$ and $\mathcal{T}_{\left.\mu\right|_{A}}$ is called the induced $\mu$-STS on $A$ from $\mathcal{T}_{\mu}$.

Theorem 1.12. Let $\left(A, \mu, \mathcal{T}_{\left.\mu\right|_{A}}\right)$ be a $\mu$-smooth subspace of $\left(X, \mu, \mathcal{T}_{\mu}\right)$ and $\lambda \in \mathcal{L}^{A}, \lambda \in[\mu]$. Then
(a) $\mathcal{F}_{\mathcal{T}_{\left.\mu\right|_{A}}}(\lambda)=\vee\left\{\mathcal{F}_{\mathcal{T}_{\mu}}(\eta): \eta \in[\mu],\left.\eta\right|_{A}=\lambda\right\}$,
(b) if $B \subset A \subset X$, then $\mathcal{T}_{\left.\mu\right|_{B}}=\left(\mathcal{T}_{\left.\mu\right|_{A}}\right)_{\left.\mu\right|_{B}}$. 
Proof.

(a) we have

$$
\begin{aligned}
\mathcal{F}_{\mathcal{T}_{\left.\mu\right|_{A}}}(\lambda) & =\mathcal{T}_{\left.\mu\right|_{A}}\left(\lambda^{\prime}\right) \\
& =\vee\left\{\mathcal{T}_{\mu}(\eta): \eta \in[\mu],\left.\eta\right|_{A}=\lambda^{\prime}\right\} \\
& =\vee\left\{\mathcal{T}_{\mu}(\eta): \eta^{\prime} \in[\mu],\left.\eta^{\prime}\right|_{A}=\lambda\right\} \\
& =\vee\left\{\mathcal{F}_{\mathcal{T}_{\mu}}\left(\eta^{\prime}\right): \eta^{\prime} \in[\mu],\left.\eta^{\prime}\right|_{A}=\lambda\right\} \\
& =\vee\left\{\mathcal{F}_{\mathcal{T}_{\mu}}(\xi): \xi \in[\mu],\left.\xi\right|_{A}=\lambda\right\} .
\end{aligned}
$$

(b) we have

$$
\begin{aligned}
\mathcal{T}_{\left.\mu\right|_{B}}(\lambda)=\vee\left\{\mathcal{T}_{\mu}(\eta): \eta \in[\mu],\left.\eta\right|_{B}=\lambda\right\} \\
=\vee\left\{\vee\left\{\mathcal{T}_{\mu}(\eta): \eta \in[\mu],\left.\eta\right|_{A}=\xi\right\}:\left.\xi \in[\mu]\right|_{A},\right. \\
\left.\left.\xi \xi\right|_{B}=\lambda\right\} \\
=\vee\left\{\mathcal{T}_{\left.\mu\right|_{A}}(\xi):\left.\xi \in[\mu]\right|_{A},\left.\xi\right|_{B}=\lambda\right\} \\
=\left(\mathcal{T}_{\left.\mu\right|_{A}}\right)_{\left.\mu\right|_{B}} .
\end{aligned}
$$

\section{Relative Smooth Continuous Maps}

The concept of continuity has been studied by Chang, Ramadan $[4,5]$ but here we shall study this concept from a different point of view.

Definition 2.1. Let $h: \mathcal{L} \rightarrow \mathcal{L}^{\prime}$ be a linear isomorphism of vector lattices (or an order preserving one-toone mapping when $\mathcal{L}$ and $\mathcal{L}^{\prime}$ are copies of $\left.[0,1]\right)$ and $\left(X, \mu, \mathcal{T}_{\mu}\right),\left(Y, \gamma, \mathcal{T}_{\gamma}\right) \mu$-STS and $\gamma$-STS, respectively. A function $f: X \rightarrow Y$ is called $(h, \mu, \gamma)$-smooth fuzzy continuous if $\mathcal{T}_{\mu}\left(f^{-1}(\eta)\right) \geq \mathcal{T}_{\gamma}(\eta)$ for all $\eta \in \mathcal{T}_{\gamma}$, where $f^{-1}(\eta)(x)=$ $(h \circ \eta)(f(x)) \cap \mu(x)$ for all $x \in X . f^{-1}(\gamma) \cap \mu$ is called the inverse image of $\gamma$ relative to $\mu$.

Remark 2.2. When $\mathcal{L}=\mathcal{L}^{\prime}=[0,1]$ and $\mu=\chi_{X}$ then the $\left(X, \chi_{X}, \tau_{\chi_{X}}\right), \chi_{X}$-RST coincides with the fuzzy topological space $(\bar{X}, \tau)$ defined by Chang [5-7].

Theorem 2.3. Let $\mathcal{L}=\mathcal{L}^{\prime}=[0,1]$ and $f: X \rightarrow Y$ be $\left(I, \chi_{X}, \chi_{Y}\right)$-fuzzy continuous, where $I: \mathcal{L} \rightarrow \mathcal{L}^{\prime}$ is the identity function. Then $f$ is continuous in Chang's view.

Proof. In Remark 2.2 we considered $\left(X, \chi_{X}, \mathcal{T}_{\chi_{X}}\right)$ and $\left(Y, \chi_{Y}, \mathcal{T}_{\chi_{Y}}\right)$ as fuzzy topological spaces. Now let $\gamma$ be an open set of smooth topology $\mathcal{T}_{\chi_{Y}}$. Then

$$
\begin{aligned}
\mathcal{T}_{\chi_{X}}\left(f^{-1}(\gamma)\right) & =\mathcal{T}_{\chi_{X}}\left(\left(I^{-1} \circ \gamma \circ f\right) \cap \chi_{X}\right) \\
& =\mathcal{T}_{\chi_{X}}(\gamma \circ f) \geq \mathcal{T}_{\chi_{X}}(\gamma) .
\end{aligned}
$$

So $f$ is a fuzzy continuous function.
Theorem 2.4. Let $\mu \in \mathcal{L}^{X}, \gamma \in \mathcal{L}^{\prime} Y$ where $\mathcal{L}$ and $\mathcal{L}^{\prime}$ are copies of $[0,1]$ and $f: X \rightarrow Y a(h, \mu, \gamma)$-fuzzy continuous functions where $f^{-1}(\gamma)=\mu$. Then for every $\gamma$-closed fuzzy set $\eta, f^{-1}(\eta)$ is a $\mu$-closed fuzzy set.

Proof. Let $\eta$ be $\gamma$-closed set. Then $\eta^{\prime}$ is a $\gamma$-open set and we have

$$
\begin{aligned}
\left(f^{-1}(\eta)\right)^{\prime} & =\mu-\inf \left\{h^{-1} \circ \eta \circ f, \mu\right\} \\
& =\sup \left\{\mu-h^{-1} \circ \eta \circ f, 0\right\} \\
& =\mu-h^{-1} \circ \eta \circ f=\mu-f^{-1}(\eta) .
\end{aligned}
$$

Hence

$$
\begin{aligned}
\mathcal{T}_{\mu}\left(f^{-1}\left(\eta^{\prime}\right)\right) & =\mathcal{T}_{\mu}\left(f^{-1}(\gamma-\eta)\right) \\
& =\mathcal{T}_{\mu}\left(h^{-1} \circ(\gamma-\eta) \circ f \cap \mu\right) \\
& =\mathcal{T}_{\mu}\left(\left(h^{-1} \circ \gamma \circ f-h^{-1} \circ \eta \circ f\right) \cap \mu\right) \\
& =\mathcal{T}_{\mu}\left(f^{-1}(\gamma)-f^{-1}(\eta)\right) \\
& =\mathcal{T}_{\mu}\left(\mu-f^{-1}(\eta)\right) \\
& =\mathcal{F}_{\mathcal{T}_{\mu}}\left(f^{-1}(\eta)\right) .
\end{aligned}
$$

So $f^{-1}(\eta)$ is a $\mu$-closed fuzzy set.

Theorem 2.5. Let $\left(X_{i}, \mu_{i}, \mathcal{T}_{\mu_{i}}\right)$ be relative smooth topological spaces for $i=1,2,3$. If $f: X_{1} \rightarrow X_{2}$ and $g: X_{2} \rightarrow X_{3}$ are relative smooth continuous maps and $\mu_{1}=f^{-1}\left(\mu_{2}\right)$ then so is $g \circ f$.

Proof. Using the relative smooth continuity of $g$ and $f$ it follows that

$$
\begin{aligned}
\mathcal{T}_{\mu_{1}}\left((g \circ f)^{-1}(\eta)\right) & =\mathcal{T}_{\mu_{1}}\left(f^{-1}\left(g^{-1}(\eta)\right)\right) \\
& \geq \mathcal{T}_{\mu_{2}}\left(g^{-1}(\eta)\right) \geq \mathcal{T}_{\mu_{3}}(\eta) .
\end{aligned}
$$

Since for every $x \in X$,

$$
\begin{aligned}
(g \circ f)^{-1}(\eta)(x) \\
\quad=\eta(g \circ f)(x) \cap \mu_{1}(x) \\
\quad=\eta(g \circ f)(x) \cap \mu_{1}(x) \cap \mu_{2}(f(x)) \\
\quad=g^{-1}\left(\eta(f(x)) \cap \mu_{1}(x)\right)=f^{-1}\left(g^{-1}(\eta)(x)\right) \\
\quad=\left(f^{-1} \circ g^{-1}\right)(\eta)(x) .
\end{aligned}
$$

Theorem 2.6. Let $\left(X, \mu, \mathcal{T}_{\mu}\right)$ and $\left(Y, \nu, \mathcal{T}_{\nu}\right)$ be two relative smooth topological spaces, $f: X \rightarrow Y$ a relative smooth continuous map, $A \subset X$, and $f^{-1}(\nu)=\mu$. Then the $\left.f\right|_{A}$ : $\left(A,\left.\mu\right|_{A}, \mathcal{T}_{\left.\mu\right|_{A}}\right) \rightarrow\left(Y, \nu, \mathcal{T}_{\nu}\right)$ is also relative smooth continuous. 
Proof. For each $\eta \in[\nu]$,

$$
\begin{aligned}
\mathcal{T}_{\left.\mu\right|_{A}}\left(\left(\left.f\right|_{A}\right)^{-1}(\eta)\right) & =\vee\left\{\mathcal{T}_{\mu}(\lambda): \lambda \in[\mu],\left.\lambda\right|_{A}=\left(\left.f\right|_{A}\right)^{-1}(\eta)\right\} \\
& \geq \mathcal{T}_{\mu}\left(f^{-1}(\eta)\right) \\
& \geq \mathcal{T}_{\nu}(\eta) .
\end{aligned}
$$

\section{The Representation of a Relative Smooth Topology}

Now we study the representation of a relative smooth topology $\mathcal{T}_{\mu}$.

Let $\left(X, \mu, \mathcal{T}_{\mu}\right)$ be a $\mu$-STS, $\alpha \in \mathcal{L}^{\prime}$. Then we define

$$
\mathcal{T}_{\mu}^{\alpha}=\left\{\lambda \in[\mu]: \mathcal{T}_{\mu}(\lambda) \geq \alpha\right\} .
$$

Theorem 3.1. Let $\left(X, \mu, \mathcal{T}_{\mu}\right)$ be a $\mu$-STS. Then for every $\alpha>$ $0, \mathcal{T}_{\mu}^{\alpha}$ is a relative topological space. Moreover $\alpha_{1} \leq \alpha_{2}$ implies $\mathcal{T}_{\mu}^{\alpha_{1}} \geq \mathcal{T}_{\mu}^{\alpha_{2}}$.

Proof. It is clear that $\chi \varnothing, \mu \in \mathcal{T}_{\mu}^{\alpha}$. When $\lambda, \eta \in \mathcal{T}_{\mu}^{\alpha}$, we have

$$
\mathcal{T}_{\mu}(\lambda) \geq \alpha, \quad \mathcal{T}_{\mu}(\eta) \geq \alpha,
$$

and so

$$
\mathcal{T}_{\mu}(\lambda \cap \eta) \geq \mathcal{T}_{\mu}(\lambda) \wedge \mathcal{T}_{\mu}(\eta) \geq \alpha
$$

This implies that $\lambda \cap \eta \in \mathcal{T}_{\mu}^{\alpha}$. When $\lambda_{j} \in \mathcal{T}_{\mu}^{\alpha}$ for each $j \in I$ we have

$$
\mathcal{T}_{\mu}\left(\cup \lambda_{j}\right) \geq \wedge \mathcal{T}_{\mu}\left(\lambda_{j}\right) \geq \alpha
$$

Hence $\cup \lambda_{j} \in \mathcal{T}_{\mu}^{\alpha}$. So $\mathcal{T}_{\mu}^{\alpha}$ is a relative topology.

The second part is trivial to verify, since for $\lambda \in \mathcal{T}_{\mu}^{\alpha_{2}}$, $\mathcal{T}_{\mu} \geq \alpha_{2} \geq \alpha_{1}, \lambda \in \mathcal{T}_{\mu}^{\alpha_{1}}$, so $\mathcal{T}_{\mu}^{\alpha_{1}} \geq \mathcal{T}_{\mu}^{\alpha_{2}}$.

Theorem 3.2. Let $\mathcal{T}_{\mu}^{\alpha}, \alpha \in(0,1]$ be a family of $\mu$-fuzzy topology on $X$ such that $\alpha_{1} \geq \alpha_{2}$ implies $\mathcal{T}_{\mu}^{\alpha_{1}} \leq \mathcal{T}_{\mu}^{\alpha_{2}}$. Let $\tau$ be the $\mathcal{L}^{\prime}$-fuzzy set built by $\tau_{\mu}(\lambda)=\vee\left\{\alpha: \lambda \in \mathcal{T}_{\mu}^{\alpha}\right\}$. Then $\tau_{\mu}$ is a $\mu$-smooth topology.

Proof.

(a) $\tau_{\mu}\left(\chi_{\varnothing}\right)=\tau_{\mu}(\mu)=1$ by the definition.

(b) For every $\lambda, \eta \in[\mu]$ and $\alpha>0$ if $\lambda, \eta \in \mathcal{T}_{\mu}^{\alpha}$ then $\lambda \cap \eta \in \mathcal{T}_{\mu}^{\alpha}$. Therefore

$$
\left\{\alpha: \lambda \cap \eta \in \mathcal{T}_{\mu}^{\alpha}\right\} \supset\left\{\alpha: \lambda \in \mathcal{T}_{\mu}^{\alpha}, \eta \in \mathcal{T}_{\mu}^{\alpha}\right\}
$$

implies that

$$
\tau_{\mu}(\lambda \cap \eta) \geq \tau_{\mu}(\lambda) \wedge \tau_{\mu}(\eta)
$$

(c) If every $\lambda_{i} \in \mathcal{T}_{\mu}^{\alpha}$ then $\cup \lambda_{i} \in \mathcal{T}_{\mu}^{\alpha}$. Since

$$
\left\{\alpha: \cup \lambda_{i} \in \mathcal{T}_{\mu}^{\alpha}\right\} \supset\left\{\alpha: \cup \lambda_{i} \in \mathcal{T}_{\mu}^{\alpha}, \forall i \in I\right\},
$$

then

$$
\tau_{\mu}\left(\cup_{i \in I} \lambda_{i}\right) \geq \wedge_{i \in I} \tau_{\mu}\left(\lambda_{i}\right)
$$

For $\tau_{\mu}$ being a relative $\mathcal{L}^{\prime}$-fuzzy set, with $\mathcal{L}^{\prime}=[0,1]$, we can state a representation theorem.

Theorem 3.3. Let $\mathcal{T}_{\mu}$ be a relative smooth topology and $\mathcal{T}_{\mu}^{\alpha}$ the $\alpha$ cut of $\mathcal{T}_{\mu}$. From the families of relative fuzzy topologies $\mathcal{T}_{\mu}^{\alpha}$ one built $\mathcal{T}_{1 \mu}(\lambda)=\vee\left\{\alpha: \lambda \in \mathcal{T}_{\mu}^{\alpha}\right\}$. Then $\mathcal{T}_{1 \mu}=\mathcal{T}_{\mu}$.

Proof. The proof is trivial from the preceding results and the well-known fact that

$$
\vee\left\{\alpha: \lambda \in \mathcal{T}_{\mu}^{\alpha}\right\}=\vee\left\{\alpha: \mathcal{T}_{\mu}(\lambda) \geq \alpha\right\}=\mathcal{T}_{\mu}(\lambda)
$$

Definition 3.4. Let $\tau$ be a Chang fuzzy topology on $X$. Then a $\mu$-smooth topology $\mathcal{T}_{\mu}$ on $X$ is said to be compatible with $\tau$ if $\tau=\left\{\lambda \in \mathcal{L}^{X}: \mathcal{T}_{\mu}(\lambda \cap \mu)>0\right\}$.

Example 3.5. Let $X$ be a nonempty set and $\mathcal{T}_{\mu}:[\mu] \rightarrow \mathcal{L}^{\prime}$ be a mapping defined by $\mathcal{T}_{\mu}(\mu)=\mathcal{T}_{\mu}\left(\chi_{\varnothing}\right)=1, \mathcal{T}_{\mu}(\lambda)=0$ for every $\lambda \in[\mu] \backslash\{\chi \varnothing, \mu\}$.

It is clear that $\mathcal{T}_{\mu}$ is the only relative smooth topology on $X$ compatible with the indiscrete fuzzy topology of Chang.

Example 3.6. Let $X$ be a nonempty set and define a mapping $\mathcal{T}_{\mu}:[\mu] \rightarrow \mathcal{L}^{\prime}$ by $\mathcal{T}_{\mu}(\mu)=\mathcal{T}_{\mu}(\chi \varnothing)=1, \mathcal{T}_{\mu}(\lambda)=\alpha$ for every $\lambda \in[\mu] \backslash\{\chi \varnothing, \mu\}$.

It is clear that $\mathcal{T}_{\mu}$ is a $\mu$-smooth topology on $X$ compatible with the discrete fuzzy topology of Chang.

\section{Relative Smooth Compactness}

Definition 4.1. Let $\left(X, \mu, \mathcal{T}_{\mu}\right)$ be a $\mu$-STS. $\lambda \in[\mu], \mathcal{A}, \mathscr{B} \subset$ $[\mu]$. $\mathcal{A}$ is called a relative cover of $\lambda$, if $\vee \mathcal{A} \geq \lambda$ particularly, $\mathcal{A}$ is called a cover $\left(X, \mu, \mathcal{T}_{\mu}\right)$ if $\mathcal{A}$ is a cover of $\mu$. $\mathcal{A}$ is called a $\mu$-open cover of $\lambda$, if $\mathcal{A}$ is a family of $\mu$-open and $\mathcal{A}$ is a cover of $\lambda$.

For a cover $\mathcal{A}$ of $\lambda, \mathscr{B}$ is called a subcover of $\lambda$, if $\mathscr{B} \subset \mathcal{A}$ and $\mathcal{B}$ is still a cover of $\lambda$.

Definition 4.2. Let $\left(X, \mu, \mathcal{T}_{\mu}\right)$ be a $\mu$-STS. For every $\alpha \in[0,1)$, a family $\mathcal{A} \subset[\mu]$ is called an $\alpha$-cover, if for every $\lambda \in$ $\mathcal{A}, \mathcal{T}_{\mu}(\lambda) \geq \alpha ; \mathcal{A}$ is called a $\mu$-open $\alpha$-cover if $\mathcal{A}$ is a family of $\mu$-open set and $\mathcal{A}$ is a $\alpha$-cover; $\mathcal{A}_{0} \subset[\mu]$ is called a sub$\alpha$-cover of $\mathcal{A}_{\text {if }} \mathcal{A}_{0} \subset \mathcal{A}$ and $\mathcal{A}_{0}$ is an $\alpha$-cover.

Definition 4.3. Let $\alpha \in[0,1)$. A $\mu$-STS $\left(X, \mu, \mathcal{T}_{\mu}\right)$ is called $\alpha$-compact if every $\mu$-open $\alpha$-cover has a finite sub- $\alpha$-cover. 
Theorem 4.4. Let $f:\left(X, \mu, \mathcal{T}_{\mu}\right) \rightarrow\left(Y, \nu, \mathcal{T}_{\nu}\right)$ be an onto $\mu$-smooth continuous mapping and $f^{-1}(\nu)=\mu$. If $\left(X, \mu, \mathcal{T}_{\mu}\right)$ is $\alpha$-compact then so is $\left(Y, \nu, \widetilde{\mathcal{T}}_{v}\right)$.

Proof. Let $\left\{\lambda_{i}: i \in I\right\}$ be a $\nu$-open $\alpha$-cover of $\nu$. Now consider the family $\left\{f^{-1}\left(\lambda_{i}\right): i \in I\right\}$, since $f$ is $\mu$-smooth continuous, we have

$$
\begin{aligned}
\lambda \in \mathcal{T}_{\nu}^{\alpha} & \Leftrightarrow \mathcal{T}_{\nu} \geq \alpha \\
& \Longleftrightarrow \mathcal{T}_{\mu}\left(f^{-1}(\lambda)\right) \geq \alpha \\
& \Longleftrightarrow f^{-1}(\lambda) \in \mathcal{T}_{\mu}^{\alpha} .
\end{aligned}
$$

It follows that $\left\{f^{-1}\left(\lambda_{i}\right): i \in I\right\}$ is a $\mu$-open $\alpha$-cover of $\mu$. Since $\left(X, \mu, \mathcal{T}_{\mu}\right)$ is $\alpha$-compact there exists a finite subset $I_{0}$ of $I$ such that $\left\{f^{-1}\left(\lambda_{i}\right): i \in I_{0}\right\}$ is a $\mu$-open $\alpha$-cover of $\left(X, \mu, \mathcal{T}_{\mu}\right)$. Since $f$ is onto, then $\left\{\lambda_{i}: i \in I_{0}\right\}$ is a $\nu$-open $\alpha$-cover of $\left(Y, \nu, \mathcal{T}_{v}\right)$, which concludes the proof.

\section{References}

[1] L. A. Zadeh, "Fuzzy sets," Information and Control, vol. 8, no. 3, pp. 338-353, 1965.

[2] M. H. Anvari and M. R. Molaei, "Genomic from the viewpoint of observational modeling," WSEAS Transactions on Biology and Biomedicine, vol. 2, no. 2, pp. 228-234, 2005.

[3] M. R. Molaei, "Relative semi-dynamical systems," International Journal of Uncertainty, Fuzziness and Knowlege-Based Systems, vol. 12, no. 2, pp. 237-243, 2004.

[4] A. A. Ramadan, "Smooth topological spaces," Fuzzy Sets and Systems, vol. 48, no. 3, pp. 371-375, 1992.

[5] C. L. Chang, "Fuzzy topological spaces," Journal of Mathematical Analysis and Applications, vol. 24, no. 1, pp. 182-190, 1968.

[6] R. Lowenl, "Fuzzy topological spaces and fuzzy compactness," Journal of Mathematical Analysis and Applications, vol. 56, no. 3, pp. 621-633, 1976.

[7] L. Ying-Ming and L. Mao-Kang, Fuzzy Topology, World Scientific, Singapore, 1997. 

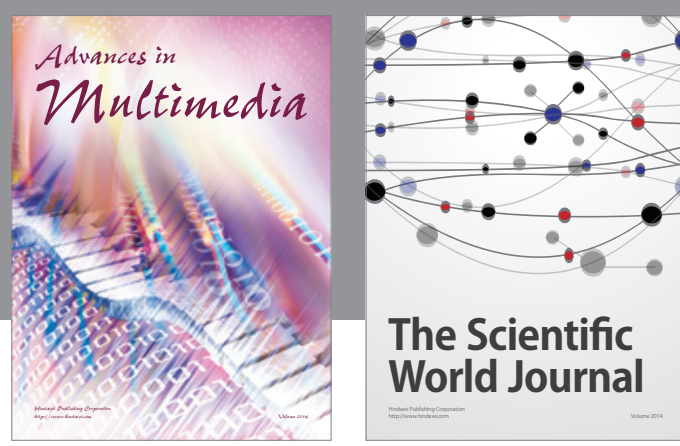

The Scientific World Journal
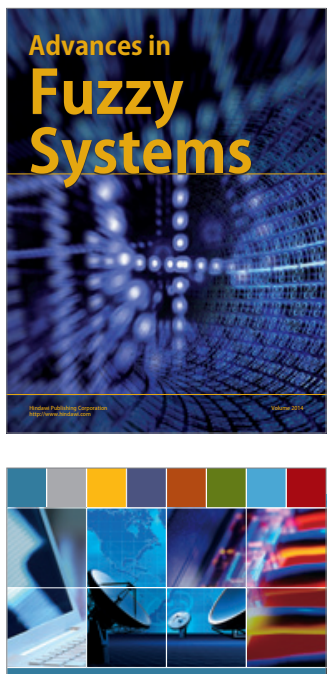

Computer Networks and Communications
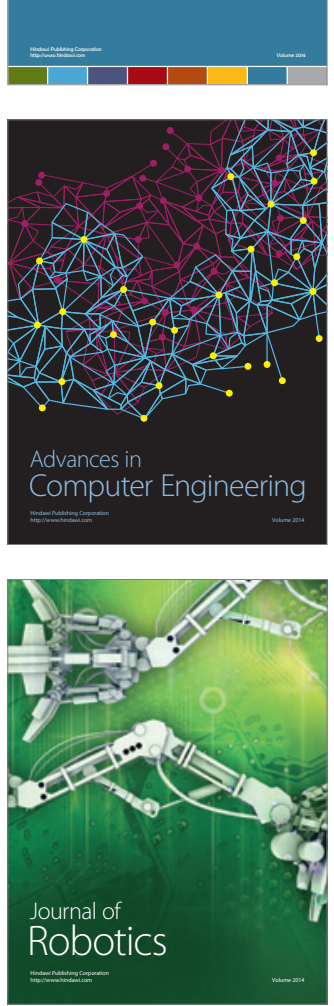
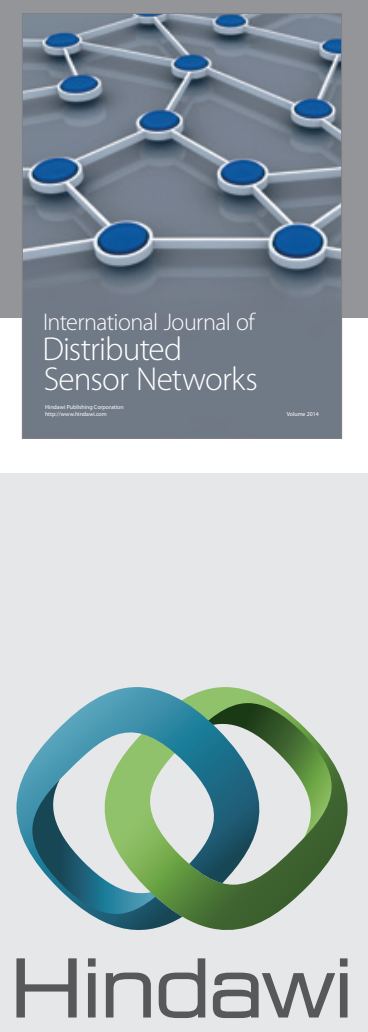

Submit your manuscripts at

http://www.hindawi.com
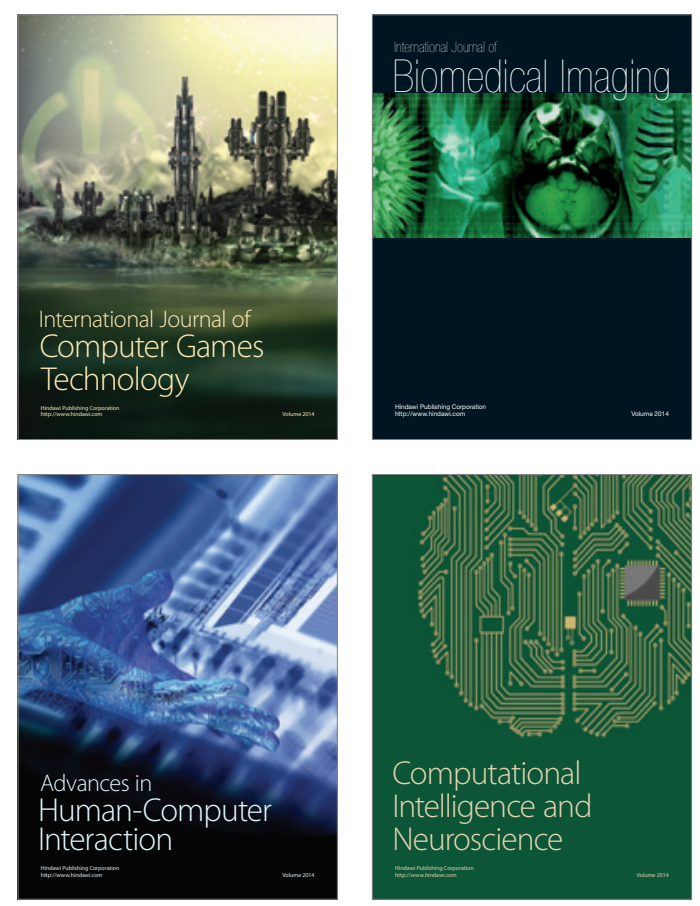
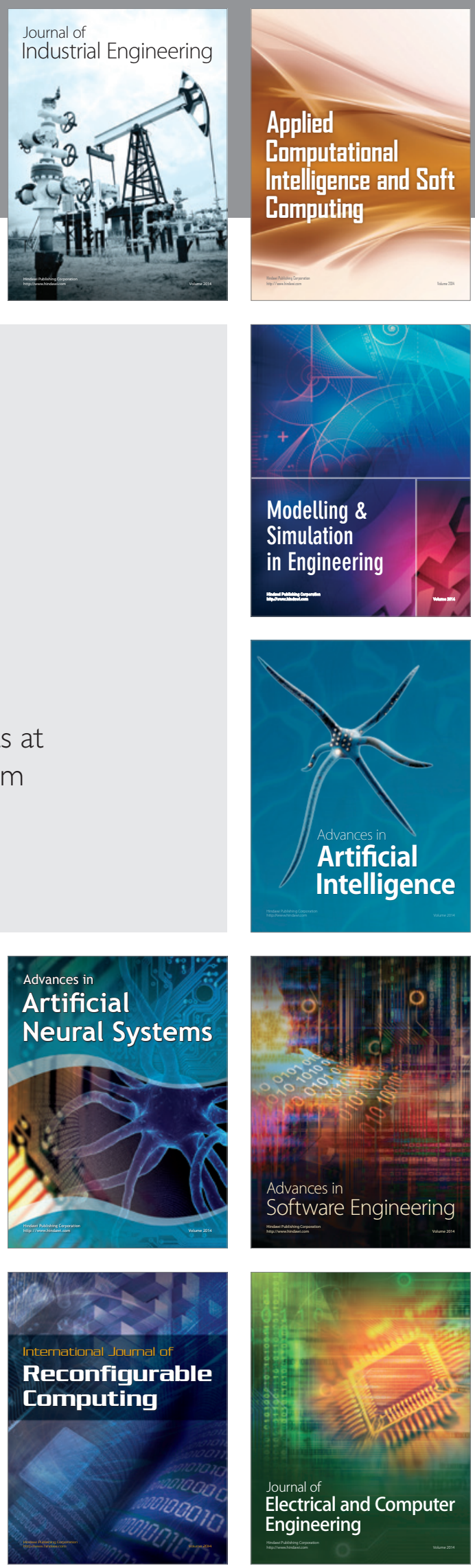